\title{
Vertrauensbasierte Organisationen als Grundlage von erfolgreichen digitalen Plattformen für personennahe Dienstleistungen
}

\author{
Carsten Schultz $(\mathbb{D}) \cdot$ Stefan Hoffmann $(\mathbb{D}) \cdot$ Manuela Ferdinand
}

Eingegangen: 13. Mai 2020 / Angenommen: 15. Juni 2020 / Online publiziert: 2. Juli 2020

(C) Der/die Autor(en) 2020

Zusammenfassung Immer mehr Unternehmen entschließen sich dazu, ihr Geschäftsmodell digital auszuweiten. Dieser Trend ist auch im Bereich der personennahen Dienstleistungen spürbar. Um Zugriff auf die digitalen Serviceangebote zu erhalten, müssen potentielle Nutzer persönliche Daten preisgeben. In vielen Fällen führt dies zu Bedenken und stellt somit ein gravierendes Hindernis für den Erfolg der Plattformen dar.

Vorliegende Studie erklärt Vertrauen als entscheidenden Faktor für den Erfolg digitaler Plattformstrategien für personennahe Dienstleistungen. Es werden Unterschiede zwischen gewinnorientierten und gemeinwohlorientierten Organisationen aufgezeigt und verdeutlicht, inwieweit eine strategische Nutzerintegration, also der Einbezug von Nutzern in den Entwicklungsprozess der Plattform und eine operative Nutzerintegration, bei der sich die Nutzer mit eigenen Ideen und Wünschen in den laufenden Betrieb der Plattform einbringen können, Einfluss auf Vertrauen und Nutzungsintention potentieller Anwender haben.

Hierzu wurde ein drei-faktorielles Online-Experiment mit den Faktoren Organisationsform, strategische Nutzerintegration und operative Nutzerintegration in einem Between-Subjects-Design durchgeführt. Es hat sich bestätigt, dass Vertrauen entscheidende Größe für die Nutzungsintention ist. Zudem hat sich herausgestellt, dass

C. Schultz

Wirtschafts- und Sozialwissenschaftliche Fakultät, Professur für Technologiemanagement, Christian-Albrechts-Universität zu Kiel, Westring 425, 24118 Kiel, Deutschland

S. Hoffmann

Wirtschafts- und Sozialwissenschaftliche Fakultät, Professur für Marketing, Christian-Albrechts-Universität zu Kiel, Olshausenstraße 40, 24098 Kiel, Deutschland

M. Ferdinand $(\bowtie)$

Wirtschafts- und Sozialwissenschaftliche Fakultät, Lehrstuhl für Technologiemanagement, Christian-Albrechts-Universität zu Kiel, Westring 425, 24118 Kiel, Deutschland

E-Mail: m.ferdinand@bwl.uni-kiel.de 
Vertrauen den Einfluss von Organisationsform und operativer Nutzerintegration auf die Nutzungsintention mediiert.

Die Ergebnisse der Studie richten sich vorrangig an Unternehmen, die sich mit der Entwicklung und Vermarktung von Plattformstrategien für personennahe Dienstleistungen beschäftigen. Solche Unternehmen erhalten hier erste wichtige Hinweise dazu, wie sie, je nach ihrer Organisationsform, diese Strategien erfolgreich umsetzen können, um so eine kritische Masse an Anwendern ihrer Plattform zu erreichen.

Schlüsselwörter Digitale Plattform · Personennahe Dienstleistung · Vertrauen · Organisationsform $\cdot$ Nutzerintegration $\cdot$ Nutzungsintention

\section{Trust Based Organisations as the Basis of Successful Digital Platforms for Personal Services}

Abstract More and more companies are deciding to digitally expand their business model. This trend is also noticeable in the area of personal services. In order to gain access to the digital service offerings, potential users must disclose personal data. In many cases, this raises concerns and therefore represents a serious obstacle to the success of the platforms.

This study explains trust as a decisive factor for the success of digital platform strategies for personal services. Differences between profit-oriented and non-profit organizations are shown and it is clarified to what extent a strategic user integration, i.e. the inclusion of users in the development process of the platform and an operational user integration, in which the users can contribute their own ideas and wishes to the ongoing operation of the platform, have an impact on the trust and intended use of potential users.

For this purpose, a three-factor online experiment was carried out with the factors organizational form, strategic user integration and operational user integration in a between-subject design. It has been confirmed that trust is a decisive factor for the intended use. It has also been shown that trust mediates the influence of organizational form and operational user integration on the intended use.

The results of the study are primarily aimed at companies that are involved in the development and marketing of platform strategies for personal services. Such companies receive initial important information on how, depending on their organizational form, they can successfully implement these strategies in order to reach a critical mass of users of their platform.

Keywords Digital platform · Personal service · Trust · Organizational form · User integration · Intended use

\section{Einleitung}

Die vorliegende Studie untersucht den Einfluss der Organisationsform und der Form der Nutzerintegration auf die Nutzungsintention digitaler Plattformen für personennahe Dienstleistungen. Digital assistierte personennahe Dienstleistungen, wie die 
Kinderbetreuung, Haushalts-, Einkaufs- und Mobilitätshilfen, haben gerade durch die aktuelle Corona Krise an Bedeutung gewonnen; werden jedoch von potentiellen Nutzern, aufgrund der notwendigen Freigabe persönlicher Daten, oftmals als risikobehaftet wahrgenommen.

Die vorliegende Untersuchung geht deshalb der Frage nach, unter welchen Bedingungen potentielle Nutzer eine lokal, im Wohnquartier angebotene, digitale Plattform nutzen würden und welche Voraussetzungen gegeben sein müssen, damit diesen digitalen Plattformen Vertrauen geschenkt wird. Ziel ist es herauszufinden, inwieweit die Faktoren „Organisationsform“, „Nutzerintegration“ und „Vertrauen“ miteinander in Zusammenhang stehen und wie sich ihr Zusammenspiel auf die Nutzungsintention personennaher digitaler Dienstleistungsplattformen auswirkt.

Hierzu wurde ein drei-faktorielles Online-Experiment mit den Faktoren Organisationsform, strategische Nutzerintegration und operative Nutzerintegration in einem Between-Subjects-Design durchgeführt. Die Ergebnisse der Untersuchung liefern wichtige Informationen für das Management zur Handhabung und Vermarktung digitaler, personenbezogener Dienstleistungsplattformen, welche durch die aktuell gestiegene Bedeutung entsprechender Dienstleistungen besondere Relevanz gewinnt.

\section{Theoretischer Hintergrund}

Dass sich digitale Geschäftsmodelle als krisenfester erweisen, wird in der fortwährenden Corona Krise deutlich. Kontaktverbote und Ausgangsbeschränkungen schränken das direkte Zusammentreffen von Angebot und Nachfrage stark ein. Virtuelle Marktplätze in Form von digitalen Plattformen gewinnen an Attraktivität. Neben kommerziellen Plattformen, entstehen vermehrt soziale Plattformen, beispielsweise digitale Nachbarschaftshilfen. Um einen wirtschaftlichen Nutzen zu erzielen, bedarf es einer kritischen Masse an Nutzern der Plattform. Je höher die Anzahl der Nutzer, desto attraktiver ist die Plattform in der Regel. Ziel eines Plattformbetreibers ist es demnach, möglichst viele potentielle Nutzer zu erreichen und diese an die Plattform zu binden.

Besonders herausfordernd ist dies in Bezug auf Dienstleistungsplattformen. Anders als bei Plattformen, bei denen es ausschließlich um Kauf und Verkauf von materiellen Produkten geht, stehen Dienstleistungen im direkten Zusammenhang mit dem Nutzer und sind nur mit diesem gemeinsam zu erbringen. Im Zuge der Corona-Krise werden vermehrt personennahe digitale Dienstleistungen angeboten. Darunter fallen beispielsweise Dienstleistungen aus den Kategorien Betreuung und Beratung (z.B. voiio.de als digitale Kinderbetreuung) sowie Pflege und Gesundheit (z.B. physioflix.de als virtueller Physiotherapeut). Allein der Bundesverband Deutscher Psychologinnen und Psychologen vermerkt auf seiner Homepage 59 zertifizierte Online-Beratungsstellen für psychische Probleme. Auch existieren diverse Plattformen (z. B. selfapy), die Personen in psychischen Belastungssituationen Unterstützung anbieten.

Damit die Betroffenen diese Unterstützung erhalten, müssen sie Nutzer der Plattform werden. Dazu ist es in der Regel notwendig, zahlreiche Angaben zur eigenen Person an den Betreiber der Plattform herauszugeben. Studien zufolge erkennen 


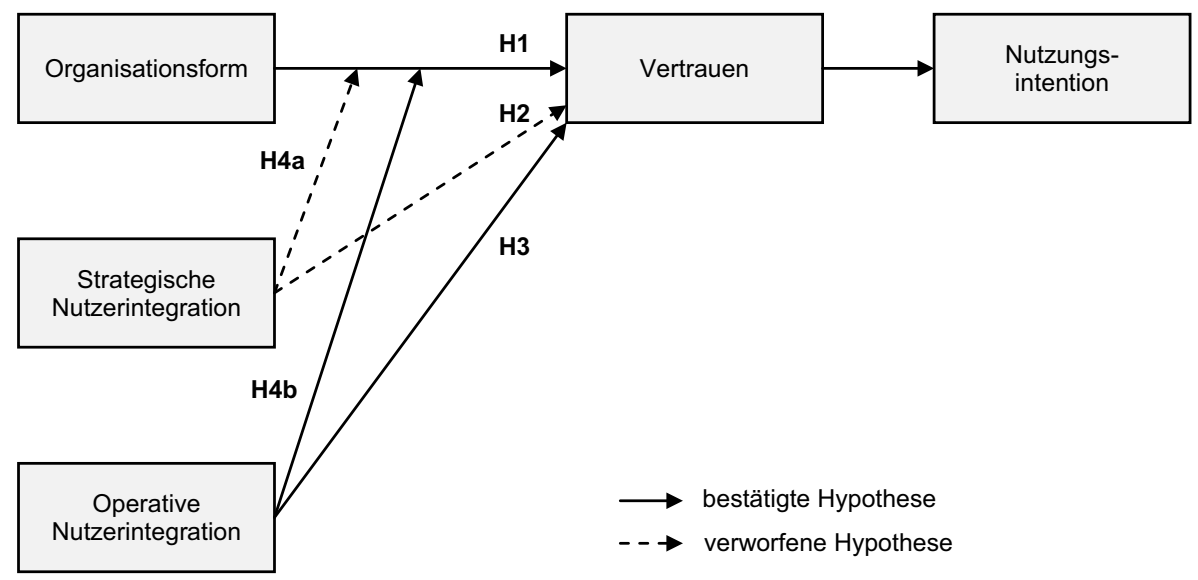

Abb. 1 Konzeptionelles Untersuchungsmodell

die meisten Menschen kein Problem darin, grundlegende soziodemografische Daten wie Alter und Geschlecht online preiszugeben. Geht es darüber hinaus (z.B. Angaben zum Beruf, Einkommen sowie die Wohnanschrift), entstehen Bedenken. Je mehr private Angaben potentielle Nutzer veröffentlichen müssen, desto stärker werden diese Bedenken in der Regel. Angaben zu Gesundheit und Finanzen werden äußerst ungern getätigt. Gemäß einer Untersuchung des GfK-Vereins (2018) geben $40 \%$ der Befragten an, Daten aus diesen Bereichen auch nicht zu veröffentlichen, wenn sie daraus einen Vorteil erlangen könnten, wie zum Beispiel einen günstigeren Tarif der Krankenkasse. Nur 12\% der Befragten geben an, gar kein Problem damit zu haben, diese Daten online bereitzustellen (GfK Verein 2017). Die vorsichtige Haltung gegenüber der Weitergabe persönlicher Daten bestätigt auch eine Untersuchung des Universitätsklinikums Freiburg. Hauptgrund, weswegen die Befragten die Nutzung von Gesundheits-Apps ablehnen, ist die Sorge um persönliche Daten. Es sei nicht möglich zu kontrollieren, was genau mit den Daten passiert und ob diese von Dritten ausgespäht werden können (Universitätsklinikum Freiburg 2013). Die Weitergabe persönlicher Daten an Online-Plattformen geht demnach mit einem gefühlten Kontrollverlust einher. Es wird ein Teil der Persönlichkeit offenbart und der Nutzer weiß nicht, zu welchem Preis dies geschieht.

Vor diesem Hintergrund untersucht die vorliegende Studie, inwiefern die Faktoren Organisationsform sowie die strategische und die operative Nutzerintegration dazu beitragen, dass Nutzer einer Plattform vertrauen und inwiefern dies die Intention, personennahe digitale Dienstleistungsplattformen zu nutzen, fördert. Abb. 1 fasst das konzeptionelle Modell der Untersuchung (Eigenentwicklung) zusammen, welches nachfolgend erläutert wird.

\subsection{Vertrauen als Mittel zur Komplexitätsreduktion}

Vertrauen ist die entscheidende Größe für die Nutzungsintention personennaher digitaler Dienstleistungsplattformen. Laut Luhmann (2014, S. 8) wirkt sich Vertrauen 
auf die wahrgenommene Komplexität einer Situation aus. Je größer das Vertrauen, desto mehr kann diese Komplexität vermindert werden. Theoretisch ist somit ein positiver Zusammenhang zwischen Vertrauen und Nutzungsintention gegeben. Welche Art von Vertrauen in Bezug auf die Nutzung digitaler Plattformen praktische Relevanz hat, muss jedoch noch weiter erforscht werden. Luhmann bezeichnet jenes Vertrauen, welches eine Person einem abstrakten Funktionssystem, also beispielsweise einer digitalen Plattform, entgegenbringt, als Systemvertrauen. Hier wird nicht in eine gewisse Leistung oder Kultur vertraut, sondern in das System als solches (Luhmann 1973). Bezieht man sich auf die Ausgangssituation (personenbezogene Daten, die in der Regel nur an vertraute Personen und Institutionen weitergegeben werden), so muss von einem reinen Systemvertrauen, wie Luhmann es definiert, abgesehen werden und tiefer in das Konstrukt des Vertrauens eingetaucht werden.

Vertrauen kann aus zwei Blickwinkeln heraus betrachtet werden: dem generalisierten Vertrauen, welches sich durch Erfahrungen über die Zeit hinweg entwickelt und dem spezifischen Vertrauen, welches sich auf eine bestimmte Person, Institution oder Situation bezieht und einstuft, ob diese vertrauenswürdig ist (Clases und Wehner 2005; Seiler 2013). Während generalisiertes Vertrauen nur schwer zu beeinflussen ist, gibt es einige Ansätze, spezifisches Vertrauen zu verstehen und zu stärken. Einige Studien betrachten die Entstehung von spezifischem Vertrauen, während andere Studien dessen Wirkung beleuchten (Kassebaum 2004). Oftmals werden die beiden Facetten affektives und kognitives Vertrauen des spezifischen Vertrauens unterschieden. Affektives Vertrauen wird auch als emotionales Vertrauen bezeichnet und basiert auf der intuitiven Einschätzung einer Person. Personen bzw. Organisationen, denen ein hohes affektives Vertrauen entgegengebracht wird, werden oftmals als nahbar, offen in der Kommunikation und wohlwollend bezeichnet (Swan et al. 1999; Gilbert 2007). Kognitives Vertrauen beruht auf systematischen Überlegungen und rationalen Entscheidungen. Unter anderem werden Werte wie Beständigkeit, Berechenbarkeit und Kompetenz mit kognitivem Vertrauen in Verbindung gesetzt (Gilbert 2007). Entsprechend dieser beiden Facetten kann davon ausgegangen werden, dass potentielle Nutzer sowohl das Wohlwollen des Betreibers (beispielsweise aus den Nutzerdaten keinen zusätzlichen Profit zu erwirtschaften) als auch das fachliche Know-How des Betreibers (beispielsweise seine Fähigkeit, eine sichere Plattform zu entwickeln) bewerten. Wir gehen ferner davon aus, dass das so entstandene Vertrauen die Intention, eine Dienstleistungsplattform zu nutzen, stark beeinflusst.

\subsection{Vertrauen und Organisationsform}

Zwischenzeitlich existiert eine Vielzahl unterschiedlicher digital vermittelter personennaher Dienstleistungen und die Plattformbetreiber stammen aus unterschiedlichen Bereichen. Grundlegend kann man zwischen gewinnorientierten und gemeinwohlorientierten Organisationen unterscheiden. Erstere streben marktwirtschaftliche Ziele wie Maximierung von Umsatz, Gewinn und Rentabilität an, während letztere ihr Handeln primär an gemeinnützigen, sozialen Aspekten ausrichten. Es stellt sich daher die Frage, ob es in der Wahrnehmung der Vertrauenswürdigkeit Unterschiede zwischen gewinnorientierten und gemeinwohlorientierten Organisationen gibt. Einzelne Untersuchungen berichten, dass die soziale Ausrichtung gemeinwohlorien- 
tierter Organisationen tendenziell einen Vertrauensvorsprung gegenüber gewinnorientierten Organisationen verschafft. Empirisch belegt ist diese Annahme beispielsweise in einer Studie der Forschungsgruppe Weltanschauung aus dem Jahr 2004. Diese offenbarte, dass Befragte der Caritas, der Diakonie und Greenpeace mehr vertrauen als etwa Krankenkassen oder politischen Institutionen. Zudem konnte im Gesundheitsektor nachgewiesen werden, dass Online-Informationen gemeinnütziger Organisationen (z. B. Universitätskliniken) eine höhere Glaubwürdigkeit erzielten als es bei gewinnorientierten Organisationen (z.B. Pharmaunternehmen) der Fall war (Held 2009, S. 26).

Während gemeinwohlorientierte Organisationen informell verbindlich ihr Handeln an gesellschaftlichen Themen ausrichten, sind solche sozialen oder ökologischen Aspekte für die meisten Wirtschaftsunternehmen eher ideelle Ziele, die neben gewinnorientierten Zielen in den betrieblichen Organisationsstrukturen festgelegt sind (Weggen 2017, S. 42).

Die soziale Ausrichtung gemeinwohlorientierter Unternehmen ist allgemein bekannt und wird in der Regel wenig hinterfragt. Ein Grund dafür kann beispielsweise das Zusammenspiel von Vertrauen und Emotionen sein. Setzen sich Organisationen aktiv für ein gesellschaftliches Anliegen ein (z. B. der NABU für die Aufrechterhaltung der Natur oder die Johanniter Unfall-Hilfe für diverse karitative und soziale Zwecke), so betrachten die meisten Menschen dies als positives Handeln. Diese Einschätzung kann dazu führen, dass gemeinnützigen Unternehmen auch weitere, positive Eigenschaften zugeschrieben werden, wie eine hohe Vertrauenswürdigkeit. Dieser sogenannte Halo-Effekt besagt, dass von bekannten Eigenschaften einer Person bzw. Organisation auf unbekannte Eigenschaften dieser geschlossen werden kann. Wirkt dieser Effekt in umgekehrter Richtung, spricht man von einem HornEffekt, der beschreibt, wie ein negativer Aspekt (so beispielsweise hohe Managementgehälter im Finanzsektor) weitere und somit auch positive Aspekte überschattet. Soziales Engagement von Wirtschaftsunternehmen steht demnach oft im Schatten dominierender Erfolgs- und Finanzziele (Weggen 2017, S. $329 \mathrm{ff}$.).

Verschafft die offensichtlich soziale Ausrichtung gemeinwohlorientierten Organisationen diesen Vertrauensvorsprung, so könnte deren Finanz- und Haushaltsplanung ein Misstrauen in die Zukunftsfähigkeit begünstigen. Gemeinwohlorientierte Organisationen sind oftmals auf Spendengelder angewiesen und werden von ehrenamtlichen Mitarbeitern geführt. Sie gelten dadurch möglicherweise als wirtschaftlich weniger beständig (Weggen 2017, S. 119f.). Es besteht somit aus Nutzersicht ein höheres Risiko, dass gemeinwohlorientierte Organisationen sich nicht lange auf einem Markt halten können. Hier profitieren gewinnorientierte Organisationen unter anderem von hochqualifiziertem Fachpersonal und verfügbarer Liquidität. Achten potentielle Nutzer besonders auf diese Faktoren, könnten auch gewinnorientierte Organisationen einen Vertrauensvorsprung vor gemeinwohlorientierten Organisationen erlangen.

Zusammengefasst gehen wir davon aus, dass die Organisationsform einen Einfluss auf das spezielle Vertrauen potentieller Nutzer einer digitalen Plattform für personennahe Dienstleistungen hat. 
H1 Die Organisationsform hat Einfluss auf das spezielle Vertrauen potentieller Nutzer.

\subsection{Vertrauen und Nutzerintegration}

Wie eingangs erläutert, kann Vertrauen dazu führen, bestehende Komplexität zu verringern. Vertrauen potentielle Nutzer, Kunden oder Geschäftspartner einer Organisation, so nehmen sie eher das Risiko einer Informationsasymmetrie in Kauf und akzeptieren mangelnde Transparenz. Eine Möglichkeit, Transparenz und demnach Vertrauen zu schaffen und direkt als Organisation davon zu profitieren, ist die Nutzerintegration. Erfolgreiche Innovationen berücksichtigen die Interessen relevanter Nutzergruppen. Die Integration potentieller Nutzer führt tendenziell zu einer höheren Innovationsgeschwindigkeit und verbesserten Qualität - letztendlich also zu einem höheren Erfolg der Innovation (Reinicke 2004, S. 23). Insbesondere personennahe digitale Dienstleistungen profitieren von den Anregungen und Ideen der Nutzer.

Grundsätzlich gibt es zwei Möglichkeiten der Nutzerintegration: Zum einen können potentielle Nutzer mit in den Entwicklungsprozess der Plattform einbezogen werden. Die Vorteile dieser strategischen Nutzerintegration bestehen in einer frühzeitigen Einbindung der relevanten Zielgruppe in den Prozess der Ideenfindung für neue oder verbesserte Lösungen. Funktions- und Designentscheidungen können in Zusammenarbeit mit potentiellen Nutzern zielgruppengerechter getroffen werden. An dieser Stelle des Innovationsprozesses macht sich vor allem die Integration sogenannter Lead User bezahlt. Darunter versteht man jene potentielle Nutzer, die über besonders relevante Fähigkeiten, Eigenschaften und Informationen verfügen, die dazu dienen, die Plattform zielgruppengerecht auszugestalten und zu vermarkten (Abele 2013). Die zweite Form der Nutzerintegration kommt dann zum Zuge, wenn die Plattform bereits in Betrieb genommen ist. Unter dieser operativen Nutzerintegration verstehen wir die Integration in den laufenden Betrieb der Plattform. Potentielle Nutzer sind demnach miteinbezogen, sich nach Inbetriebnahme der Plattform gemeinsam zu entscheiden, wie die Plattform im Verlauf gestaltet wird; beispielsweise welche Dienstleister in die Plattform aufgenommen werden und welche persönlichen Daten an wen weitergegeben werden. Zudem übernehmen die Nutzer eine Aufsichtsfunktion gegenüber den Betreibern und stellen die kollektiven Interessen aller Nutzer sicher.

Wir vermuten daher, dass sich die Nutzerintegration, unabhängig von der Organisationsform, positiv auf das spezielle Vertrauen und die Nutzungsintention auswirkt.

H2 Die strategische Nutzerintegration in die Entwicklung der Plattform wirkt sich positiv auf das spezielle Vertrauen potentieller Anwender aus.

H3 Die operative Nutzerintegration in den laufenden Betrieb der Plattform wirkt sich positiv auf das spezielle Vertrauen potentieller Anwender aus.

Es wird im Weiteren davon ausgegangen, dass etwaige, durch die gewinn- oder gemeinwohlorientierte Organisationsausrichtung, bestehende Vertrauensdefizite, durch stattfindende Nutzerintegration kompensiert werden können. Da die Einflüsse der 
Interaktion zwischen Organisationsform und Nutzerintegration auf die Nutzungsintention durch das Vertrauen mediiert werden, wirkt sich die Interaktion auch positiv auf die Nutzungsintention aus.

H4a Defizite im speziellen Vertrauen von gemeinwohlorientierten Organisationen werden durch die strategische/operative Nutzerintegration abgeschwächt.

H4b Defizite im speziellen Vertrauen von gewinnorientierten Organisationen werden durch die strategische/operative Nutzerintegration abgeschwächt.

\section{Stichprobe und Aufbau des Fragebogens}

Zur Überprüfung der Hypothesen wurde eine quantitative, experimentelle OnlineBefragung durchgeführt. Die Befragung erfolgte in Kooperation mit einem PanelDienstleister, sodass ein repräsentatives Abbild der Bevölkerung angestrebt werden konnte. Wie eingangs angebracht, wird der Frage nachgegangen, unter welchen Bedingungen potentielle Nutzer eine lokal im Wohnquartier angebotene digitale Plattform nutzen würden und welche Voraussetzungen gegeben sein müssen, damit diesen digitalen Plattformen Vertrauen geschenkt wird. Zielgruppe der Befragung stellten demnach Bewohner von Mietwohnungen in Deutschland dar. Da die Plattform auf lokalen Dienstleistungsangeboten basiert, wurde als Einschlusskriterium definiert, dass der Wohnort der Befragten mindestens 2000 Einwohner umfasst. Zudem mussten die Befragten ein Smartphone oder ein Tablet besitzen, welches sie im Alltag nutzen.

Im Befragungszeitraum, Februar 2020, konnten insgesamt 352 Mieter erreicht werden; davon 64\% Frauen und 36\% Männer. Das Durchschnittsalter liegt bei 45,2 Jahren. Die Mehrheit der Befragten lebt entweder mit ihrem Partner zusammen $(40 \%)$ oder alleine $(37 \%)$. Etwas über die Hälfte der Befragten $(53 \%)$ gibt an, Kinder zu haben; davon überwiegend ein Kind. Die Anzahl der Befragten, die pflegebedürftige Angehörige im Haushalt betreuen, liegt bei $4 \%$. Die Mehrheit von $62 \%$ der Befragten lebt im städtischen Umfeld; $28 \%$ davon in einer Stadt mit mehr als 500.000 Einwohnern. Fast die Hälfte aller Befragten (47\%) gibt an, in Vollzeit berufstätig zu sein.

Grundelement des Fragebogens stellen die Textvignetten dar, die experimentell variiert wurden (siehe Abschn. 4: Untersuchungsdesign und methodisches Vorgehen, Anhang A). In verschiedenen, voneinander abweichenden Textbausteinen werden Organisationsform und Nutzerintegration erläutert und der Befragte soll darauf bezogen verschiedene Aussagen bewerten.

Der sich daran anschließende Fragebogen enthält Aussagen zur Nutzungsintention; also der Absicht, die digitale Plattform unter den in der Vignette angebrachten Bedingungen nutzen zu wollen (eigene Formulierung in Anlehnung an Tan et al. (2007): „Ich finde das Angebot allgemein attraktiv“, ,Ich finde das Angebot auf mich bezogen attraktiv“, „Ich würde das Angebot annehmen“, „Wenn das Angebot in der Vergangenheit bereits existiert hätte, hätte ich es genutzt", Cronbachs alpha =0,942, $\mathrm{M}=2,74, \mathrm{SD}=1,12)$. Die Aussagen zur Nutzungsintention, wie auch die Items aller 
anderen Variablen, wurden auf einer fünfstufigen Ratingskala ( 1 = stimmt gar nicht, $5=$ stimmt völlig) abgefragt.

Das spezielle Vertrauen wurde in Anlehnung an Barchard (2001), durch eigene Itemformulierungen erfasst (,Anhand objektiver Kriterien komme ich zu dem Schluss, dass der Betreiber der LivingSmart App vertrauenswürdig ist“”, „Nach einer strukturierter Bewertung schätze ich die Vertrauenswürdigkeit des Betreibers der LivingSmart App hoch ein“, „Anhand rationaler Aspekte komme ich zu dem Entschluss, dass der Betreiber der LivingSmart App vertrauenswürdig ist“, „Mein Instinkt sagt mir, dass ich dem Betreiber der LivingSmart App vertrauen kann“, „Meinem „Bauchgefühl“ zufolge ist der Betreiber der LivingSmart App vertrauenswürdig"). Die Items umfassen Aspekte des affektiven und kognitiven Vertrauens. Wie eine explorative Faktorenanalyse zeigt, fallen diese jedoch auf einen Faktor zusammen und werden im Folgenden unter dem Faktor ,spezielles Vertrauen“ zusammengefasst (Cronbachs alpha $=0,972, \mathrm{M}=3,09, \mathrm{SD}=0,83$ ).

Als Kontrollvariablen wurden noch die folgenden Skalen abgefragt: Interpersonales Vertrauen (Beierlein et al. 2012; z.B. „Ich bin davon überzeugt, dass die meisten Menschen gute Absichten haben“), Technikaffinität (Neyer et al. 2012; z.B. „Hinsichtlich technischer Neuerungen bin ich sehr neugierig“), Einstellung zu Digitalen Dienstleistungen (Janssen 2000; z. B. „In meinem Umfeld bin ich gewöhnlich der Erste, der neue, digitale Dienstleistungen nutzt"), Persönlichkeitseigenschaften (Rammstedt et al. 2014; Big-5 Persönlichkeitsfaktoren: Neurotizismus, Extraversion, Offenheit für Erfahrung, Verträglichkeit, Gewissenhaftigkeit), Risikobereitschaft (Sharma 2010; z. B. „Ich ziehe eine routinierte Lebensweise einer unbeständigen Lebensweise vor"). Zudem wurden die Befragten nach ihren Erfahrungen mit digitalen Dienstleistungen in verschiedenen Kategorien befragt. Darunterfallen: Einkauf, Mobilität, Bildung, Gesundheit, Instandhaltung, Betreuung, Finanzen und Verwaltung, soziale Integration und sonstige Services.

\section{Untersuchungsdesign und methodische Vorgehensweise}

Die Studie basiert auf einem drei-faktoriellen experimentellen Design mit den Faktoren Organisationsform, strategische Nutzerintegration und operative Nutzerintegration. In einem Between-Subjects-Design wurde jeder Teilnehmer zu Beginn des Online-Fragebogens gebeten, eine Textvignette zu lesen, die randomisiert aus einem Set von acht Vignetten gezogen wurde (Anhang A und B). Die Textvignetten enthalten hypothetische Beschreibungen der fiktiven „LivingSmart“-App, die sich systematisch durch die Kombination verschiedener Ausprägungen der drei Merkmale Organisationsform (gemeinwohlorientiert, gewinnorientiert), strategische Nutzerintegration und operative Nutzerintegration unterscheiden (siehe Tab. 1).

Folglich handelt es sich in dieser Studie um ein $2 \times 2 \times 2$-faktorielles experimentelles Design, welches den Einfluss von drei unabhängigen Variablen (Organisationsform, strategische Nutzerintegration, operative Nutzerintegration) auf die abhängigen Variablen (Nutzungsintention, Vertrauen) untersucht. Im Rahmen eines Between Subject Designs erhält jeder Teilnehmer per Zufall nur eine Experimentalbedingung, um Ermüdungserscheinungen vorzugreifen. Zudem verringert dies die 
Tab. 1 Vignetten

\begin{tabular}{lll}
\hline Vignette & Organisationsform & Nutzerintegration \\
\hline 1 & Gemeinwohlorientiert & Strategisch, operativ \\
2 & Gemeinwohlorientiert & Strategisch \\
3 & Gemeinwohlorientiert & Operativ \\
4 & Gemeinwohlorientiert & - \\
5 & Gewinnorientiert & Strategisch, operativ \\
6 & Gewinnorientiert & Strategisch \\
7 & Gewinnorientiert & Operativ \\
8 & Gewinnorientiert & - \\
\hline
\end{tabular}

Wahrscheinlichkeit, dass Befragte die Zielsetzung der Untersuchung antizipieren und ihr Antwortverhalten bewusst in eine bestimmte Richtung ausrichten. Der anschließende Fragebogen war für alle Befragten inhaltlich gleich aufgebaut.

Um die Hypothesen zu prüfen, wurden mehrfaktorielle Varianzanalysen mittels SPSS durchgeführt. Im ersten Schritt wurden die direkten Effekte der unabhängigen Variablen auf das spezielle Vertrauen untersucht. Um unbeobachtete Varianz auszuschließen, wurden die Kontrollvariablen Geschlecht, Technikaffinität und Einstellung zur Digitalisierung analysiert. Keine dieser Variablen hat einen Einfluss auf die Haupteffekte im Modell ausgeübt. Somit sind keine Verzerrungen in den Ergebnissen zu erwarten.

\section{Ergebnisse}

Übergreifend konnte nachgewiesen werden, dass eine gemeinwohlorientierte Organisationsausrichtung mit einem höheren speziellen Vertrauen und einer höheren Nutzungsintention einhergeht. Die mehrfaktorielle Varianzanalyse bestätigt, dass die Gewinnorientierung einen negativen Einfluss auf das Vertrauen potentieller Nutzer hat $(p=0,012)$. Hypothese 1 kann bestätigt werden.

Deutlich wird der Unterschied zwischen gemeinwohlorientierten und gewinnorientierten Organisationsformen auch in der deskriptiven Auswertung. Über alle Untersuchungsgruppen hinweg liegt der Mittelwert der Nutzungsintention bei 2,75 (SD: 1,12). Betrachtet man die Mittelwerte der einzelnen Gruppen, so ist zu erkennen, dass die Mittelwerte von gemeinwohlorientierten Organisationen, ausgenommen der ersten Manipulation, durchgehend höher sind als die von gewinnorientierten Organisationen (siehe Abb. 2).

Zudem wurde festgestellt, dass sich die operative Nutzerintegration positiv auf die Nutzungsintention und das spezielle Vertrauen auswirkt. Auch wirkt sich die Interaktion von Organisationsform und operativer Nutzerintegration stark signifikant auf die Nutzungsintention ( $p=0,003)$ aus (siehe Abb. 3, links).

Es besteht kein signifikanter Einfluss der strategischen Nutzerintegration auf das spezielle Vertrauen potentieller Nutzer $(p=0,705)$; die operative Nutzerintegration hingegen wirkt sich positiv auf das spezielle Vertrauen aus $(p=0,027)$. Während Hypothese 2 verworfen werden muss, kann Hypothese 3 angenommen werden. 


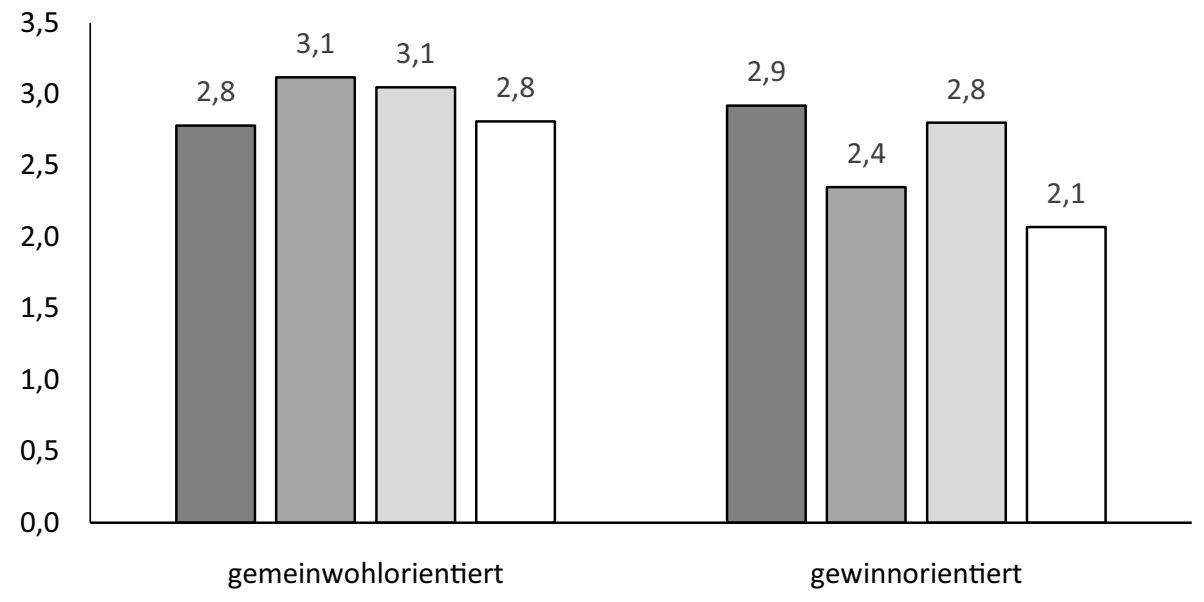

$\square$ operativ, strategisch $\square$ strategisch $\quad$ operativ $\square$ nicht eingebunden

Abb. 2 Nutzungsintention in Abhängigkeit von der experimentellen Manipulation
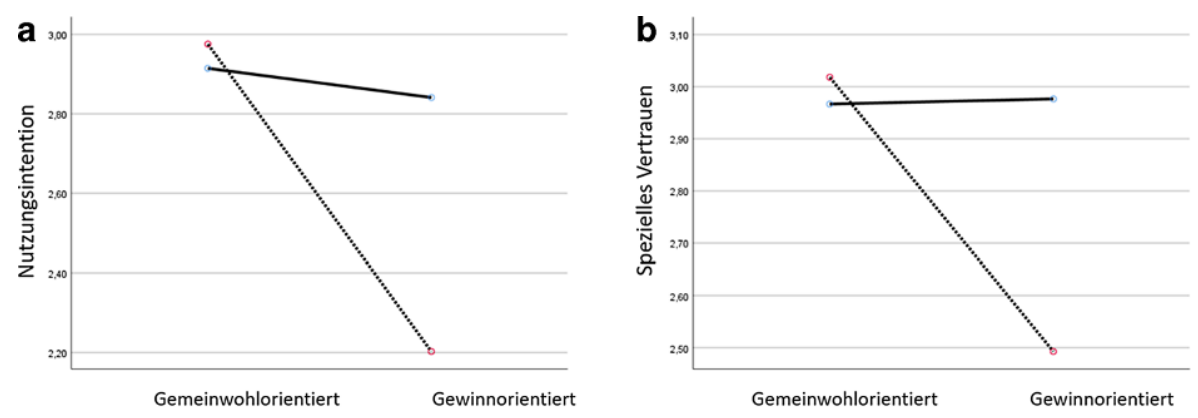

$-\ldots$ Operative Nutzerintegration inklusive
Operative Nutzerintegration exklusive

Abb. 3 Interaktion von operativer Nutzerintegration und Organisationsform auf die Nutzungsintention und das spezielle Vertrauen

Aufgrund des nicht signifikanten Einflusses der strategischen Nutzerintegration, wird im Folgenden nur die operative Nutzerintegration auf ihre kompensierende Wirkung hin näher analysiert. Demnach muss Hypothese 4a verworfen werden $(p=0,698)$. Die Integration potentieller Nutzer in den Betrieb (operative Nutzerintegration) kann bestehende Vertrauensdefizite bei gemeinwohlorientierten Organisationen nicht kompensieren. Hingegen kann Hypothese $4 \mathrm{~b}$ angenommen werden. Es besteht ein hoch signifikanter Effekt der Interaktion zwischen Organisationsform und operativer Nutzerintegration auf das spezielle Vertrauen $(p=0,009)$, wie auch grafisch veranschaulicht in Abb. 3 (rechts) zu erkennen ist.

Während gemeinwohlorientierte Organisationen in Bezug auf das spezielle Vertrauen nicht von einer operativen Nutzerintegration profitieren, profitieren gewinnorientierte Organisation stark von dieser. Gewinnorientierte Organisationen können 
durch eine stattfindende operative Nutzerintegration ,attraktiver“ wirken, bzw. von der Nutzerintegration stärker profitieren, um das Interesse potentieller Nutzer zu wecken. Von einer strategischen Nutzerintegration profitiert hingegen keine der beiden Organisationsformen.

Schließlich wurde untersucht, ob der Faktor Vertrauen den Einfluss der Interaktion von Organisationsform und operativer Nutzerintegration mediiert. Berechnet wurde dies mit Hilfe des SPSS-Makro PROCESS, welches es ermöglicht, mediationsspezifische Zusatzergebnisse zu ermitteln. Zur Berechnung wurden bootstrap-basierte Signifikanztests zu den indirekten Effekten durchgeführt. Auf Grundlage vorab bestimmter Vertrauenswahrscheinlichkeiten wurden für die indirekten Effekte Konfidenzintervalle ermittelt. Ein signifikanter Mediatoreffekt kann dann bestätigt werden, wenn dieses Konfidenzintervall nicht den Wert Null beinhaltet (Hayes 2013). Dies war in dieser Berechnung der Fall ( $p=0,008, \mathrm{LLCI}=-0,972, \mathrm{ULCI}=-0,144)$. Der Faktor des speziellen Vertrauens fungiert als vollständiger Mediator und stellt somit den Zusammenhang zwischen der Interaktion von Organisationsform und operativer Nutzerintegration und der Nutzungsintention her. Das bedeutet, dass das spezielle Vertrauen von der Unternehmensform und operativer Nutzerintegration abhängig ist, beide aber keinen, über das spezielle Vertrauen hinausgehenden, Effekt auf die Nutzungsintention besitzen.

\section{Zusammenfassung und Diskussion}

Die vorliegende Studie gibt Aufschluss darüber, welche Einflussfaktoren relevant sind, um potentielle Anwender dazu zu bewegen, digitale Plattformen für personennahe Dienstleistungen zu nutzen. Erstmals wurden drei Aspekte (Organisationsform, Nutzerintegration, Vertrauen) zusammengeführt und aufgezeigt, wie diese miteinander agieren und die Nutzung der Plattformen beeinflussen.

Es hat sich herausgestellt, dass gewinnorientierte Organisationen tendenziell einen negativen Einfluss auf das spezielle Vertrauen haben (H1). Durch die Einbindung potentieller Nutzer kann diesem negativen Einfluss entgegengewirkt werden. Hier hat sich die operative Nutzerintegration als eine sehr wertbringende Maßnahme herausgestellt (H3), während sich die strategische Nutzerintegration sowohl bei gewinnorientierten als auch bei gemeinwohlorientierten Organisationen als nicht relevant erwiesen hat. Die Interaktion zwischen der Organisationsform und der operativen Nutzerintegration hat sich auch, speziell für gewinnorientierte Organisationen, als sehr relevant herausgestellt. Gewinnorientierte Organisationsformen profitieren stark von der Einbindung möglicher Nutzer in den laufenden Betrieb der Plattform (H4b). Gemeinwohlorientierte Unternehmen erhalten dadurch hingegen keinen zusätzlichen Vorteil - ihr wohlwollendes Image trägt dazu bei, dass ihnen generell stärker vertraut wird.

Die Studie bestätigt die Mediatorfunktion von Vertrauen. Ein höheres Vertrauen erklärt die Einflüsse, sowohl der Organisationsform, als auch der operativen Nutzerintegration, auf die Nutzungsintention vollständig. Vertrauen kann demnach als zentrale Größe für den Erfolg von digitalen Plattformstrategien für personennahe Dienstleistungen angesehen werden. 


\subsection{Limitationen und weitere Forschung}

Auch wenn vorliegende Studie einige zentrale und valide Ergebnisse liefern konnte, bedarf es weiterer Forschungsaktivitäten. Die zukünftige Forschung sollte stärker auf Unterschiede zwischen verschiedenen Dienstleistungen wie Kinderbetreuung, Haushaltshilfen und Mobilität eingehen. Zudem ist es notwendig, nicht nur potentielle Nutzergruppen aus Sicht der Dienstleistungsnehmer (in dieser Studie Bewohner einer Mietwohnung) zu untersuchen, sondern auch Dienstleistungsgeber mit in die Studie zu integrieren. Es muss herausgefunden werden, wie Dienstleister auf unterschiedliche Organisationsformen reagieren, wie diese in die Entwicklung und den Betrieb der Plattform integriert werden müssen und inwieweit dort Vertrauen gebildet wird. Zuletzt ist anzumerken, dass Experimente eine begrenzte ökologische Validität haben. Um auch die ökologische Validität zu erhöhen, sollte zukünftig eine Feldstudie in einem vorab definierten Wohnquartier durchgeführt werden, um die hier gewonnenen Resultate unter realen Bedingungen zu überprüfen.

\subsection{Implikationen für die Praxis}

Unabhängig davon, wie gut die Technik und wie vielfältig das Angebot ist - um eine digitale Plattform für personennahe Dienstleistungen erfolgreich auf dem Markt positionieren zu können, bedarf es einer kritischen Masse an Nutzern dieser Plattform. Unternehmen stehen vor der Herausforderung, potentielle Nutzer von ihrer Geschäftsidee zu überzeugen. Die Ergebnisse dieser empirischen Studie zeigen deutlich, dass es Unterschiede in der Wahrnehmung zwischen gemeinwohlorientierten und gewinnorientierten Organisationsformen gibt. Somit gibt es nicht einen goldenen Weg die kritische Nutzermasse zu erreichen, sondern organisationsspezifische Herangehensweisen. Zentrale Einflussgröße bleibt aber in jedem Fall das Vertrauen. Hier hat sich gezeigt, dass gemeinwohlorientierten Organisationen generell ein höheres Vertrauen zugewiesen wird als gewinnorientierten Organisationen. Während gewinnorientierte Organisationen demnach zusätzlich in den Faktor Vertrauen investieren sollten, ist dieser Schritt für gemeinwohlorientierte Organisationen weniger relevant. Als vertrauensfördernde Maßnahme sollten gewinnorientierte Organisationen sich mit den Möglichkeiten einer operativen Nutzerintegration auseinandersetzen. Nutzer sollten durch institutionelle Regeln ein Auskunfts- und Mitgestaltungsrecht an der Plattform eingeräumt werden. Dadurch näheren sich beide Organisationsformen einander an und es werden auch spezifische Ausprägungen wie Genossenschaften denkbar.

Förderung Diese Arbeit wurde durch das Bundesministerium für Bildung und Forschung (BMBF) gefördert (Förderkennzeichen: 02K17A051) und vom Projektträger Karlsruhe (PTKA) betreut.

Funding Open Access funding provided by Projekt DEAL.

Open Access Dieser Artikel wird unter der Creative Commons Namensnennung 4.0 International Lizenz veröffentlicht, welche die Nutzung, Vervielfältigung, Bearbeitung, Verbreitung und Wiedergabe in jeglichem Medium und Format erlaubt, sofern Sie den/die ursprünglichen Autor(en) und die Quelle ordnungsgemäß nennen, einen Link zur Creative Commons Lizenz beifügen und angeben, ob Änderungen vorgenommen wurden. 
Die in diesem Artikel enthaltenen Bilder und sonstiges Drittmaterial unterliegen ebenfalls der genannten Creative Commons Lizenz, sofern sich aus der Abbildungslegende nichts anderes ergibt. Sofern das betreffende Material nicht unter der genannten Creative Commons Lizenz steht und die betreffende Handlung nicht nach gesetzlichen Vorschriften erlaubt ist, ist für die oben aufgeführten Weiterverwendungen des Materials die Einwilligung des jeweiligen Rechteinhabers einzuholen.

Weitere Details zur Lizenz entnehmen Sie bitte der Lizenzinformation auf http://creativecommons.org/ licenses/by/4.0/deed.de.

\section{Anhang A}

\section{Vignette 1 (Beispiel)}

Betreiber der App ist der Verein für innovative Quartiersarbeit. Als gemeinnütziger Verein setzt sich dieser für ein nachhaltiges Wohnen ein. Mit der Einführung der App erwartet der Verein eine zunehmende Zufriedenheit der Bewohner sowie ein besseres Zusammenleben unter sozialen und umweltbezogenen Aspekten. Die App soll den Bewohnern kostenfrei und funktionsfähig in vollem Umfang zur Verfügung gestellt werden.

In der Entwicklungsphase unterstützen die Bewohner die Mitglieder des Vereins für innovative Quartiersarbeit bei der Gestaltung der App.

Die Bewohner sind aktiv einbezogen gemeinsam zu entscheiden in welcher Form Dienstleistungen in die APP integriert werden und welche persönlichen Daten der Bewohner dazu benötigt werden und in welcher Form die Bewohner davon profitieren. 


\section{Anhang B}

Tab. B.1 Systematische Variation der Textvignetten

\begin{tabular}{|c|c|c|}
\hline $\begin{array}{l}\text { Organisa- } \\
\text { tionsform }\end{array}$ & $\begin{array}{l}\text { Betreiber der App ist der Verein für in- } \\
\text { novative Quartiersarbeit. Als gemein- } \\
\text { nütziger Verein setzt sich dieser für ein } \\
\text { nachhaltiges Wohnen ein. Mit der Ein- } \\
\text { führung der App erwartet der Verein eine } \\
\text { zunehmende Zufriedenheit der Bewohner } \\
\text { sowie ein besseres Zusammenleben unter } \\
\text { sozialen und umweltbezogenen Aspekten. } \\
\text { Die App soll den Bewohnern kostenfrei } \\
\text { und funktionsfähig in vollem Umfang zur } \\
\text { Verfügung gestellt werden }\end{array}$ & $\begin{array}{l}\text { Betreiber der App ist die Bau und Wohn } \\
\text { GmbH. Als gewinnorientiertes Wirt- } \\
\text { schaftsunternehmen setzt sich das Unter- } \\
\text { nehmen für Bau, Bewirtschaftung, Ver- } \\
\text { waltung und Vermarktung von Immobili- } \\
\text { en ein. Die Einführung der App begründet } \\
\text { das Unternehmen mit einer prognosti- } \\
\text { zierten Zunahme der Zufriedenheit von } \\
\text { Bewohnern sowie als Werbemaßnahme } \\
\text { für weitere Mietinteressenten }\end{array}$ \\
\hline $\begin{array}{l}\text { Strategische } \\
\text { Integration }\end{array}$ & $\begin{array}{l}\text { In der Entwicklungsphase unterstützen } \\
\text { die Bewohner die Mitglieder des Vereins } \\
\text { für innovative Quartiersarbeit bei der } \\
\text { Gestaltung der App }\end{array}$ & $\begin{array}{l}\text { In der Entwicklungsphase sind aus- } \\
\text { schließlich Mitarbeiter des Vereins für } \\
\text { innovative Quartiersarbeit in die Gestal- } \\
\text { tung der App involviert }\end{array}$ \\
\hline $\begin{array}{l}\text { Operative } \\
\text { Integration }\end{array}$ & $\begin{array}{l}\text { Der Verein für innovative Quartiersar- } \\
\text { beit entscheidet allein, in welcher Form } \\
\text { Dienstleistungen in die APP integriert } \\
\text { werden und welche persönlichen Daten } \\
\text { der Bewohner dazu benötigt werden und } \\
\text { in welcher Form die Bewohner davon } \\
\text { profitieren }\end{array}$ & $\begin{array}{l}\text { Die Bewohner sind aktiv einbezogen ge- } \\
\text { meinsam zu entscheiden in welcher Form } \\
\text { Dienstleistungen in die APP integriert } \\
\text { werden und welche persönlichen Daten } \\
\text { der Bewohner dazu benötigt werden und } \\
\text { in welcher Form die Bewohner davon } \\
\text { profitieren }\end{array}$ \\
\hline
\end{tabular}




\section{Literatur}

Abele T (2013) Suchfeldbestimmung und Ideenbewertung. In: Abele T (Hrsg) Einführung in die Suchfeldbestimmung und Ideenbewertung in der frühen Phase des Innovationsprozesses. Springer, Wiesbaden, S $1-18$

Barchard KA (2001) Emotional and Social Intelligence: Examining Its Place in the Nomological Network. Faculty of Graduate Studies, University of British Columbia. https://doi.org/10.14288/1.0090848

Beierlein C, Kemper CJ, Kovaleva A., Rammstedt B (2012) Kurzskala zur Messung des zwischenmenschlichen Vertrauens: die Kurzskala Interpersonales Vertrauen (KUSIV3). (GESIS-Working Papers, 2012/22). Mannheim: GESIS - Leibniz-Institut für Sozialwissenschaften. https://nbn-resolving.org/ urn:nbn:de:0168ssoar-312126

Clases C, Wehner T (2005) Vertrauen in Wirtschaftsbeziehungen. In: Frey D, v. Rosenstiel L, Graf Hoyos C (Hrsg) Handbuch Wirtschaftspsychologie. Beltz, Weinheim, S 397-401

GfK Verein (2017) Studie „Deutsche behalten persönliche Daten lieber für sich“. https://www.gfk. com/de/insights/press-release/deutsche-behalten-persoenliche-daten-lieber-fuer-sich/. Zugegriffen: 06.05.2020

Gilbert DU (2007) Vertrauen als Gegenstand der ökonomischen Theorie. Z Manag 2:60

Hayes AF (2013) Introduction to mediation, moderation, and conditional process analysis: a regression-based approach. Guilford, New York

Held G (2009) Online-Shopping. Einflussfaktoren auf Auswahl- und Nutzungsentscheidungen von Electronic-Commerce-Anwendungen im Dienstleistungsbereich. Dissertation. Wirtschafts- und Sozialwissenschaftliche Fakultät der Universität Rostock, Rostock

Janssen O (2000) Job demands, perceptions of effort-reward fairness and innovative work behaviour. J Occup Organ Psychol 73:287-302

Kassebaum U (2004) Interpersonelles Vertrauen. Entwicklung eines Inventars zur Erfassung spezifischer Aspekte des Konstrukts. Dissertation. Wirtschafts- und Sozialwissenschaftliche Fakultät der Universität Hamburg, Hamburg

Luhmann N (2014) Vertrauen. Ein Mechanismus der Reduktion sozialer Komplexität. 5. UTB, Stuttgart

Luhmann N (1973) Vertrauen. Ein Mechanismus der Reduktion sozialer Komplexität. 2. UTB, Stuttgart

Neyer FJ, Felber J, Gebhardt C (2012) Entwicklung und Validierung einer Kurzskala zur Erfassung von Technikbereitschaft. Diagnostica 58:87-99

Rammstedt B, Kemper CJ, Klein MC, Beierlein C, Kovaleva A (2014) Big Five Inventory (BFI-10), S 23

Reinicke T (2004) Möglichkeiten und Grenzen der Nutzerintegration in der Produktentwicklung - eine Systematik zur Anpassung von Methoden zur Nutzerintegration. Dissertation. TU Berlin, Berlin

Seiler R (2013) Die Auswirkungen der Vertrauenswürdigkeit eines Online-Shops auf die Ziele des eCRM. Wirtschafts- und Sozialwissenschaftlichen Fakultät der Universität Bern, Bern

Sharma P (2010) Measuring personal cultural orientations: scale development and validation. J Acad Mark Sci 38(6):787-806

Swan JE, Bowers MR, Richardson LD (1999) Customer trust in the salesperson: An integrative review and meta-analysis of the empirical literature. J Bus Res 44(2):93-107

Tan DS, Smith G, Lee B, Robertson GG (2007) Adaptivitree: adaptive tree visualization for tournamentstyle brackets. IEEE Trans Visual Comput Graph 13(6):1113-1120

Universitätsklinikum Freiburg (2013) Gesundheits- und Versorgungs-Apps. Hintergründe zu deren Entwicklung und Einsatz. Studienzentrum Freiburg, Freiburg

Weggen J (2017) Vertrauen, Solidarität und Emotionen in Non-Profit-Organisationen: eine soziologische Analyse des Dritten Sektors in Deutschland. Hamburg University Press, Hamburg 